\title{
Integrable magnetic geodesic flows on 2-torus: new example via quasi-linear system of PDEs.
}

\author{
S.V. Agapov1, M. Bialy², A.E. Mironov ${ }^{1}$
}

\begin{abstract}
The only one example has been known of magnetic geodesic flow on the 2-torus which has a polynomial in momenta integral independent of the Hamiltonian. In this example the integral is linear in momenta and corresponds to a one parametric group preserving the Lagrangian function of the magnetic flow. In this paper the problem of integrability on one energy level is considered. This problem can be reduced to a remarkable Semihamiltonian system of quasi-linear PDEs and to the question of existence of smooth periodic solutions for this system. Our main result states that the pair of Liouville metric with zero magnetic field on the 2-torus can be analytically deformed to a Riemannian metric with small magnetic field so that the magnetic geodesic flow on an energy level is integrable by means of a quadratic in momenta integral. Thus our construction gives a new example of smooth periodic solution to the Semi-hamiltonian (Rich) quasilinear system of PDEs.
\end{abstract}

Keywords: Rich quasi-linear systems, Semi-Hamiltonian systems, Magnetic geodesic flow, Polynomial integrals.

\section{Introduction and main results}

Consider Hamiltonian system with two degrees of freedom

$$
\dot{x}^{j}=\left\{x^{j}, H\right\}, \quad \dot{p}_{j}=\left\{p_{j}, H\right\}, \quad j=1,2
$$

\footnotetext{
${ }^{1}$ Sobolev Institute of Mathematics, Novosibirsk, Russia. Supported by RSF (grant 1411-00441)

${ }^{2}$ School of Mathematical Sciences, Raymond and Beverly Sackler Faculty of Exact Sciences, Tel Aviv University, Israel. Supported by ISF grant 162/15.
} 
with a Hamiltonian function $H$. Let us remind, that the Hamiltonian system is called integrable if there exists a function (the first integral) $F\left(x_{1}, x_{2}, p_{1}, p_{2}\right)$, such that

$$
\dot{F}=\{F, H\}=\sum_{i=1}^{2}\left(\frac{\partial F}{\partial x^{i}} \frac{\partial H}{\partial p_{i}}-\frac{\partial F}{\partial p_{i}} \frac{\partial H}{\partial x^{i}}\right)=0
$$

and $F$ is functionally independent with $H$ almost everywhere.

Geodesic flow of a Riemannian metric $d s^{2}=g_{i j} d x^{i} d x^{j}$ on the 2-torus can be viewed as a Hamiltonian system with Hamiltonian function $H=\frac{1}{2} g^{i j} p_{i} p_{j}$. On the 2-torus there are two known types of metrics with integrable geodesic flow. Namely, in the first case the metric in some coordinates has the form

$$
d s^{2}=\Lambda\left(x^{1}\right)\left(\left(d x^{1}\right)^{2}+\left(d x^{2}\right)^{2}\right)
$$

In this case there exists an additional integral which is linear in momenta

$$
F=p_{2}
$$

In the second case the metric is Liouville, that is in some coordinates it has the form

$$
d s^{2}=\left(\Lambda_{1}\left(x^{1}\right)+\Lambda_{2}\left(x^{2}\right)\right)\left(\left(d x^{1}\right)^{2}+\left(d x^{2}\right)^{2}\right) .
$$

Then there exists an integral quadratic in momenta

$$
F=\frac{\Lambda_{2}\left(x^{2}\right) p_{1}^{2}-\Lambda_{1}\left(x^{1}\right) p_{2}^{2}}{\Lambda_{1}\left(x^{1}\right)+\Lambda_{2}\left(x^{2}\right)}
$$

The question of existence of metrics on the 2-torus with polynomial first integral of higher degree is old and turns out to be very complicated (see [5], [6], [7], [14], [18], [20]).

The question goes back to G. Birkhoff [9], who completed the study integrals of degree 1 and 2, and in more modern time it was conjectured in [11] that the two cases described above exhaust all examples of integrable geodesic flows on the 2-torus. This conjecture is not known even for polynomial integrals of degrees 3,4. In our recent papers (see [4]- [7]) we invented new approach to the problem and proved in particular that it is equivalent to the problem of existence of smooth periodic solutions of a system of quasi-linear PDEs of the form (3) (see below) which turns out to be semi-Hamiltonian (or Rich). Moreover in some cases using [22, the behavior of smooth solutions and their blow up can be understood for these quasi-linear systems [3], [7], [8]. 
Hamiltonian system of magnetic geodesic flow is described by the same Hamiltonian function $H=\frac{1}{2} g^{i j} p_{i} p_{j}$, but with the symplectic structure twisted by the 2 -form of the magnetic field $\Omega=\Omega\left(x_{1}, x_{2}\right) d x_{1} \wedge d x_{2}$. Thus in the magnetic case the Poisson bracket takes the form:

$$
\begin{gathered}
\{F, H\}_{m g}= \\
=\sum_{i=1}^{2}\left(\frac{\partial F}{\partial x^{i}} \frac{\partial H}{\partial p_{i}}-\frac{\partial F}{\partial p_{i}} \frac{\partial H}{\partial x^{i}}\right)+\Omega\left(x^{1}, x^{2}\right)\left(\frac{\partial F}{\partial p_{1}} \frac{\partial H}{\partial p_{2}}-\frac{\partial F}{\partial p_{2}} \frac{\partial H}{\partial p_{1}}\right) .
\end{gathered}
$$

Many questions related to integrability of magnetic flows have been considered in the literature ( [2], [10], [12], [13], [15], [24], [25]). The following, is the only one known example of polynomially integrable magnetic geodesic flows on 2torus with non-zero magnetic field:

Example 1. Let the Riemannian metric be of the form

$$
d s^{2}=\Lambda(y)\left(d x^{2}+d y^{2}\right),
$$

and the magnetic form

$$
\Omega=-u^{\prime}(y) d x \wedge d y .
$$

Then the first integral is linear in momenta and takes the form

$$
F_{1}=p_{1}+u(y)
$$

It is plausible that no other examples of integrable magnetic flows exist on the 2-torus. We shall prove this guess in Section 4 for quadratic in momenta integrals. The underlying idea here is very natural: the dynamics of magnetic flow is changing if the energy levels of $H$ varies. So the requirement for $F$ to be the first integral on all levels is a heavy requirement. However, the proof of this fact in the general case is not known and might be complicated.

Therefore, we turn now to the much more interesting problem of existence of Polynomial integral on a particular level $\left\{H=\frac{1}{2}\right\}$. It turns out, that the problem of existence of integrable magnetic flows on one energy level can be reduced to a fundamental question on existence of smooth periodic solutions of a quasi-linear PDEs of the following form:

$$
A(U) U_{x}+B(U) U_{y}=0,
$$


where the matrices $A(U), B(U)$ depend explicitly on the components of the vector $U=\left(u_{1}, \ldots, u_{n}\right)^{T}$ containing the metric factor $\Lambda$ and some coefficients of the polynomial integral $F$.

The system of the form (3) appears for integrable geodesic flows and magnetic geodesic flows. It was derived in [4 - 6] and proved that (3) has infinitely many conservation laws and in the hyperbolic region it can be written in the form of Riemann invariants. Such systems are called Semihamiltonian systems of hydrodynamic type (or Rich). They were introduced in [26], [27] (see also in [22]). We will discuss the properties of (3) in Section 2 in more details.

Let us notice that if the magnetic field is equal to zero identically, then for $n=2$, the system (3) is linearly degenerate and this property of the system enables the existence of smooth periodic solutions which give rise to the first integral of the form (1). When the magnetic field is present, it turns out that system (3) is not linearly degenerate anymore and moreover the sign of the derivatives $\frac{\partial \lambda_{i}}{\partial r_{i}}$ changes along characteristics, where $r_{i}$ is the Riemann invariant, $\lambda_{i}$ is the corresponding eigenvalue, so the question of existence of smooth periodic solutions becomes very complicated. The main challenge here is to prove or disprove the existence of periodic solutions of these systems. The main result of the present paper is that for the case of quadratic integral, i.e. $n=2$, and the system is $4 \times 4$, we managed to overcome these difficulties to get:

Theorem 1. There exist real analytic Riemannian metrics on the 2-torus which are arbitrary close to the Liouville metrics (and different from them) and a non-zero analytic magnetic fields such that magnetic geodesic flows on the energy level $\left\{H=\frac{1}{2}\right\}$ have polynomial in momenta first integral of degree two.

Let us stress again that our theorem gives a very natural $4 \times 4$ Rich quasi-linear system which is not linearly degenerate having analytic periodic solutions. We refer to [16] for other examples of a very different nature.

The idea of the proof of the main theorem is as follows. Using the specific form of the matrices of the system (3) for $n=2$, we introduce another quasi-linear system of evolution equations (formula (11) below) which form a symmetry of our system (3). The flow of this system acts on the space of solutions. Moreover, we choose the Liouville metric together with the zero magnetic field as the initial data of this flow. Then this flow for any small $t>0$ generates the nontrivial Riemannian metrics and a non-zero magnetic 
field which satisfy the requirements of Theorem 1. It is remarkable fact, which is not hard to verify, that the set of "symmetric" solutions with respect to this symmetry consists only of flat metrics with zero magnetic fields so does not produce any interesting example.

\section{Semi-hamiltonian systems of hydrodynamic type}

Systems like (3) arise when geodesic flows are studied. Such systems have many beautiful properties.

It is shown in [5] that in the case of the integrable geodesic flow one may introduce global semi-geodesic coordinates $(t, x)$ on the 2 -torus such that

$$
d s^{2}=g^{2}(t, x) d t^{2}+d x^{2}, \quad H=\frac{1}{2}\left(\frac{p_{1}^{2}}{g^{2}}+p_{2}^{2}\right) .
$$

The condition $\dot{F}=\{F, H\}=0$, where the first integral $F$ has the form

$F=\frac{a_{0}}{g^{n}} p_{1}^{n}+\frac{a_{1}}{g^{n-1}} p_{1}^{n-1} p_{2}+\ldots+\frac{a_{n-2}}{g^{2}} p_{1}^{2} p_{2}^{n-2}+\frac{a_{n-1}}{g} p_{1} p_{2}^{n-1}+a_{n} p_{2}^{n}, a_{k}=a_{k}(t, x)$

is equivalent to the system of quasi-linear PDEs of the form (3) with $U=$ $\left(a_{0}, \ldots, a_{n-2}, a_{n-1}\right)^{T}, a_{n-1}=g, a_{n}=1$. In this case $A$ is the identity matrix and

$$
B=\left(\begin{array}{cccccc}
0 & 0 & \ldots & 0 & 0 & a_{1} \\
a_{n-1} & 0 & \ldots & 0 & 0 & 2 a_{2}-n a_{0} \\
0 & a_{n-1} & \ldots & 0 & 0 & 3 a_{3}-(n-1) a_{1} \\
\ldots & \ldots & \ldots & \ldots & \ldots & \ldots \\
0 & 0 & \ldots & a_{n-1} & 0 & (n-1) a_{n-1}-3 a_{n-3} \\
0 & 0 & \ldots & 0 & a_{n-1} & n a_{n}-2 a_{n-2}
\end{array}\right)
$$

This system has many interesting properties. It can be written in the form of conservation laws, i.e. there exists a change of variables $U^{T} \rightarrow\left(Q_{1}(U), \ldots, Q_{n}(U)\right)$ such that for some $R_{1}(U), \ldots, R_{n}(U)$ the following relations hold:

$$
\left(Q_{j}(U)\right)_{t}+\left(R_{j}(U)\right)_{x}=0, \quad j=1, \ldots, n .
$$

Moreover, in the hyperbolic region, where all the eigenvalues $\lambda_{1}, \ldots, \lambda_{n}$ of matrix $B$ are real and pairwise distinct the system possesses $n$ Riemann's 
invariants $r_{1}(U), \ldots, r_{n}(U)$ such that the system can be written in the following form:

$$
\left(r_{j}\right)_{t}+\lambda_{j}(r)\left(r_{j}\right)_{x}=0, \quad j=1, \ldots, n .
$$

Such systems are called semi-hamiltonian (see [5]). Semi-hamiltonian systems were introduced and studied by S.P. Tsarev in [26], 27] (see also [22], 23]).

The same question but in conformal coordinates $d s^{2}=\Lambda(x, y)\left(d x^{2}+d y^{2}\right)$ was studied in [6]. Given a polynomial integral of the form

$$
F=a_{0} p_{1}^{n}+a_{1} p_{1}^{n-1} p_{2}+\ldots+a_{n} p_{2}^{n}, \quad a_{k}=a_{k}(x, y)
$$

it then follows from Kolokoltsov's theorem (see [18]) that the following relations hold true

$$
a_{n}=c_{1}+a_{n-2}-a_{n-4}+\ldots, \quad a_{n-1}=c_{2}+a_{n-3}-a_{n-5}+\ldots,
$$

where $c_{1}, c_{2}$ are some constants. The rest of unknown coefficients and the conformal factor satisfy the quasi-linear system of PDEs of the form (3), where $U=\left(a_{0}, \ldots, a_{n-2}, \Lambda\right)^{T}$. In this case this system also turns out to be semi-hamiltonian (in the regions where at least one of the matrixes $A$ and $B$ is non-degenerate).

For any semi-hamiltonian system the following relations on the eigenvalues hold:

$$
\partial_{r_{j}} \frac{\partial_{r_{i}} \lambda_{k}}{\lambda_{i}-\lambda_{k}}=\partial_{r_{i}} \frac{\partial_{r_{j}} \lambda_{k}}{\lambda_{j}-\lambda_{k}}, \quad i \neq j \neq k \neq i .
$$

It means that there is a diagonal metric

$$
d s^{2}=H_{1}^{2}(r) d r_{1}^{2}+\ldots+H_{n}^{2}(r) d r_{n}^{2}
$$

with Christoffel symbols satisfying the following relations:

$$
\Gamma_{k i}^{k}=\frac{\partial_{r_{i}} \lambda_{k}}{\lambda_{i}-\lambda_{k}}, \quad i \neq k
$$

It is proved in [6] that in the case of conformal coordinates the rotation coefficients $\beta_{k l}$ of the metric (4) associated with the system (3) are symmetric:

$$
\beta_{k l}=\beta_{l k}, \quad \beta_{k l}=\frac{\partial_{r_{k}} H_{l}}{H_{k}}, \quad k \neq l .
$$

This means that there is a function $a(r)$ such that $\partial_{r_{k}} a(r)=H_{k}^{2}(r)$. Here $H_{i}$ are Lame coefficients of the metric (4), $H_{i}^{2}=g_{i i}$. Such metrics are called 
the metrics of Egorov type. Following [21], we shall call the corresponding semi-hamiltonian systems to be of Egorov type.

As it is shown in [21], if the system does not split $\left(\partial_{r_{i}} \lambda_{k} \not \equiv 0, i \neq k\right)$, then it is of Egorov type iff it possesses two special conservation laws:

$$
P_{x}+Q_{y}=0, \quad P_{y}+R_{x}=0 .
$$

In the case of conformal coordinates such conservation laws were found explicitly for the system (3) in [6].

Similar results hold for the magnetic geodesic flow on the 2-torus. Such flows (or, equivalently, systems with gyroscopic forces) were studied, for example, in [10], [19], [24], [25]. In conformal coordinates the existence of an additional polynomial in momenta first integral of an arbitrary degree $N$ on the fixed energy level $\left\{H=\frac{1}{2}\right\}$ leads to the PDEs of the form (3) which is also proved to be semi-hamiltonian (see 4]). Moreover, in 44 it's proved that in the case of $N=2,3$ this is the Egorov type system. Recently this result was generalized to the case of an arbitrary degree $N$ (see [1]).

\section{The proof of the theorem}

Consider Hamiltonian system

$$
\dot{x}^{j}=\left\{x^{j}, H\right\}_{m g}, \quad \dot{p}_{j}=\left\{p_{j}, H\right\}_{m g}, \quad j=1,2
$$

on a 2 -torus in the presence of magnetic field with Hamiltonian $H=\frac{1}{2} g^{i j} p_{i} p_{j}$ and the Poisson bracket of the form (2). Choose the conformal coordinates $(x, y)$ in which

$$
d s^{2}=\Lambda(x, y)\left(d x^{2}+d y^{2}\right), \quad H=\frac{p_{1}^{2}+p_{2}^{2}}{2 \Lambda}
$$

Then one can parametrize the momenta on the energy level $\left\{H=\frac{1}{2}\right\}$ in the following way:

$$
p_{1}=\sqrt{\Lambda} \cos \varphi, \quad p_{2}=\sqrt{\Lambda} \sin \varphi .
$$

The equations (5) take the form

$$
\dot{x}=\frac{\cos \varphi}{\sqrt{\Lambda}}, \quad \dot{y}=\frac{\sin \varphi}{\sqrt{\Lambda}}, \quad \dot{\varphi}=\frac{\Lambda_{y}}{2 \Lambda \sqrt{\Lambda}} \cos \varphi-\frac{\Lambda_{x}}{2 \Lambda \sqrt{\Lambda}} \sin \varphi-\frac{\Omega}{\Lambda} .
$$


Following [4, we shall search for the first integral $F$ of the second degree in the following way:

$$
F(x, y, \varphi)=\sum_{k=-2}^{k=2} a_{k}(x, y) e^{i k \varphi}, \quad a_{k}=u_{k}+i v_{k}, \quad a_{-k}=\bar{a}_{k} .
$$

The condition $\dot{F}=0$ is equivalent to the following equation:

$$
F_{x} \cos \varphi+F_{y} \sin \varphi+F_{\varphi}\left(\frac{\Lambda_{y}}{2 \Lambda} \cos \varphi-\frac{\Lambda_{x}}{2 \Lambda} \sin \varphi-\frac{\Omega}{\sqrt{\Lambda}}\right)=0 .
$$

Let's substitute (6) into (7) and equate the coefficients at $e^{i k \varphi}$ to zero. We obtain

$$
\begin{gathered}
\frac{\Lambda_{y}}{2 \Lambda} \frac{i(k-1) a_{k-1}+i(k+1) a_{k+1}}{2}-\frac{\Lambda_{x}}{2 \Lambda} \frac{i(k-1) a_{k-1}-i(k+1) a_{k+1}}{2 i}+ \\
+\frac{\left(a_{k-1}\right)_{x}+\left(a_{k+1}\right)_{x}}{2}+\frac{\left(a_{k-1}\right)_{y}-\left(a_{k+1}\right)_{y}}{2 i}-\frac{i k \Omega a_{k}}{\sqrt{\Lambda}}=0,
\end{gathered}
$$

where $k=0, \ldots, 3$, and $a_{k}$ are assumed to be zero when $k>2$. For $k=3$, we get from (8):

$$
\left(a_{2}\right)_{x}-\frac{\Lambda_{x}}{\Lambda} a_{2}+\frac{1}{i}\left(\left(a_{2}\right)_{y}-\frac{\Lambda_{y}}{\Lambda} a_{2}\right)=0 .
$$

Multiplying this identity by $\Lambda^{-1}$ we obtain:

$$
\left(a_{2} \Lambda^{-1}\right)_{x}-i\left(a_{2} \Lambda^{-1}\right)_{y}=0 .
$$

Thus $a_{2} \Lambda^{-1}$ is anti-holomorphic function on 2-torus and hence must be constant. Consequently $a_{2}=(\alpha+i \beta) \Lambda$ for some non-zero constant $\alpha+$ $i \beta$. Applying appropriate rotation in the plane $(x, y)$ and dividing $F$ by appropriate constant we can assume that $\alpha=1, \beta=0$. Thus we get $a_{2}=\Lambda$. Moreover, multiplying by $\Lambda^{-\frac{1}{2}}$ the imaginary part of (8) for $k=2$ we compute:

$$
\Omega=\frac{1}{4}\left(\left(v_{1} \Lambda^{-\frac{1}{2}}\right)_{x}-\left(u_{1} \Lambda^{-\frac{1}{2}}\right)_{y}\right) .
$$

Let us introduce new functions:

$$
f=\frac{u_{1}}{\sqrt{\Lambda}}, \quad g=\frac{v_{1}}{\sqrt{\Lambda}} .
$$


Then, we get the following system of equations on the unknowns $\Lambda, u_{0}, f, g$ :

$$
\begin{gathered}
f_{x}+g_{y}=0, \\
(f \Lambda)_{x}-(g \Lambda)_{y}=0, \\
\left(u_{0}\right)_{x}+2 \Lambda_{x}-\frac{1}{2} g\left(f_{y}-g_{x}\right)=0, \\
-\left(u_{0}\right)_{y}+2 \Lambda_{y}+\frac{1}{2} f\left(f_{y}-g_{x}\right)=0,
\end{gathered}
$$

which is equivalent to the matrix form:

$$
A(U) U_{x}+B(U) U_{y}=0,
$$

where

$$
A=\left(\begin{array}{cccc}
0 & 0 & 1 & 0 \\
f & 0 & \Lambda & 0 \\
2 & 1 & 0 & \frac{g}{2} \\
0 & 0 & 0 & -\frac{f}{2}
\end{array}\right), \quad B=\left(\begin{array}{cccc}
0 & 0 & 0 & 1 \\
-g & 0 & 0 & -\Lambda \\
0 & 0 & -\frac{g}{2} & 0 \\
2 & -1 & \frac{f}{2} & 0
\end{array}\right)
$$

$U=\left(\Lambda, u_{0}, f, g\right)^{T}$. The magnetic field takes the form

$$
\Omega=\frac{1}{4}\left(g_{x}-f_{y}\right),
$$

hence the magnetic form is exact. One can check that

$$
U_{0}(x, y)=\left(\begin{array}{c}
\Lambda_{1}(x)+\Lambda_{2}(y) \\
2 \Lambda_{2}(y)-2 \Lambda_{1}(x) \\
0 \\
0
\end{array}\right)
$$

is the solution of the system (9), where $\Lambda_{1}(x)$ and $\Lambda_{2}(y)$ are periodic positive functions: $\Lambda_{1}(x+1)=\Lambda_{1}(x), \Lambda_{2}(y+1)=\Lambda_{2}(y)$. This solution corresponds to the case of geodesic flow of the Liouville metric with zero magnetic field having the first integral (1). In the sequel we assume $\Lambda_{1}$ and $\Lambda_{2}$ to be real analytic functions.

We introduce the following evolution equations which are explicitly written in the form:

$$
U_{t}=A_{1}(U) U_{x}+B_{1}(U) U_{y},
$$


where

$$
A_{1}=\left(\begin{array}{cccc}
g & 0 & 0 & \Lambda \\
-2 g & g & 0 & -2 \Lambda \\
0 & 0 & 0 & 0 \\
0 & -2 & 0 & 0
\end{array}\right), \quad B_{1}=\left(\begin{array}{cccc}
f & 0 & \Lambda & 0 \\
2 f & f & 2 \Lambda & 0 \\
0 & 2 & 0 & 0 \\
0 & 0 & 0 & 0
\end{array}\right)
$$

It turns out that this system of evolution equations define the symmetry of the system (9) so that the flow of (11) transforms solutions to solutions as we shall prove below.

Next we apply the following consequence of Cauchy-Kowalevskaya theorem:

Lemma 1. The Cauchy problem for the system (11) with the initial data

$$
\left.U(x, y, t)\right|_{t=0}=U_{0}(x, y)
$$

has a unique analytic periodic $(U(x+1, y, t)=U(x, y+1, t)=U(x, y, t))$ solution for $t$ small enough.

Proof. Since the entries of the matrices $A(U), B(U)$ are linear functions in the components of $U$, it follows from Cauchy-Kowalevskaya theorem (see [17]) that there exists an analytic solution $U(x, y, t)$ of the system (11) defined on a domain $D$ containing the plane $\{t=0\}$. Moreover, since the initial data of (11) is chosen to be 1-periodic in $x, y$ it follows from the uniqueness part of the theorem that the domain $D$ can be chosen invariant under the 1-shifts $x \rightarrow x+1$ and $y \rightarrow y+1$ and the solution $U(x, y, t)$ is 1 -periodic in $x, y$. This proves Lemma 1.

Let us prove that $U(x, y, t)$ constructed in Lemma 1 is a solution of our system (9) for all small $t$. We denote by $\tilde{V}(x, y, t)$ the following real analytic vector function

$$
\tilde{V}=A(U) U_{x}+B(U) U_{y}
$$

By our construction $\tilde{V}(x, y, 0)=0$. We have to prove that $\tilde{V} \equiv 0$. Denote

$$
\tilde{V}=\left(\begin{array}{c}
V_{1} \\
V_{2} \\
V_{3} \\
V_{4}
\end{array}\right), \quad V=\left(\begin{array}{c}
V_{2} \\
V_{3} \\
V_{4}
\end{array}\right)
$$


By direct calculations using (11) one can check that $\tilde{V}$ satisfies the following system of equations:

$$
\left(\begin{array}{l}
V_{1} \\
V_{2} \\
V_{3} \\
V_{4}
\end{array}\right)_{t}=A_{2}\left(\begin{array}{l}
V_{1} \\
V_{2} \\
V_{3} \\
V_{4}
\end{array}\right)_{x}+B_{2}\left(\begin{array}{l}
V_{1} \\
V_{2} \\
V_{3} \\
V_{4}
\end{array}\right)_{y}+C_{2}\left(\begin{array}{l}
V_{1} \\
V_{2} \\
V_{3} \\
V_{4}
\end{array}\right)+D_{2}\left(\begin{array}{c}
V_{1}^{2} \\
V_{1} V_{2} \\
V_{1} V_{3} \\
V_{2} V_{3}
\end{array}\right) .
$$

Here

$$
\begin{array}{cc}
A_{2}=\left(\begin{array}{cccc}
0 & 0 & 0 & 0 \\
-g \Lambda & g & 0 & -2 \Lambda \\
0 & 0 & 0 & 0 \\
2 \Lambda & -2 & f & g
\end{array}\right), & B_{2}=\left(\begin{array}{cccc}
0 & 0 & 0 & 0 \\
f \Lambda & f & 2 \Lambda & 0 \\
2 \Lambda & 2 & f & g \\
0 & 0 & 0 & 0
\end{array}\right), \\
C_{2}=\left(\begin{array}{cccc}
0 & 0 & 0 & 0 \\
c_{1} & c_{2} & c_{3} & -2 \Lambda_{x} \\
c_{4} & 0 & f_{y} & -f_{x} \\
c_{5} & 0 & -g_{y} & g_{x}
\end{array}\right), & D_{2}=\left(\begin{array}{cccc}
0 & 0 & 0 & 0 \\
-\frac{f \Lambda}{g} & -\frac{f}{g} & -\frac{4 \Lambda}{g} & -\frac{4}{g} \\
0 & 0 & 0 & 0 \\
0 & 0 & 0 & 0
\end{array}\right),
\end{array}
$$

where

$$
\begin{gathered}
c_{1}=\frac{2 \Lambda f_{x} f+\left(f^{2}-g^{2}+4 \Lambda\right) \Lambda_{x}+2 \Lambda u_{0_{x}}}{g}, c_{2}=\frac{2\left(g g_{x}+2 \Lambda_{x}+u_{0_{x}}\right)}{g}, \\
c_{3}=\frac{4 \Lambda f_{x}+2 f \Lambda_{x}}{g}, c_{4}=4 \Lambda_{y}+\frac{1}{2} f\left(f_{y}-g_{x}\right), c_{5}=4 \Lambda_{x}-\frac{1}{2} g\left(f_{y}-g_{x}\right) .
\end{gathered}
$$

So we have

$$
V_{1_{t}}=0
$$

hence

$$
V_{1}(x, y, t)=0 .
$$

Taking this into account, we can simplify the system (13). Let us write it down explicitly:

$$
\begin{gathered}
V_{2_{t}}=\frac{2\left(g g_{x}+2 \Lambda_{x}+u_{0_{x}}\right)}{g} V_{2}+\frac{4 \Lambda f_{x}+2 f \Lambda_{x}}{g} V_{3}-\frac{4}{g} V_{2} V_{3}+g V_{2_{x}}-2 \Lambda V_{4_{x}}+ \\
+f V_{2_{y}}+2 \Lambda V_{3_{y}}-2 \Lambda_{x} V_{4} \\
V_{3_{t}}=2 V_{2_{y}}+f V_{3_{y}}+g V_{4_{y}}+f_{y} V_{3}-f_{x} V_{4}
\end{gathered}
$$




$$
V_{4_{t}}=-2 V_{2_{x}}+f V_{3_{x}}+g V_{4_{x}}-g_{y} V_{3}+g_{x} V_{4} .
$$

Let us remark here that $g \equiv 0$ for $t=0$ and therefore Cauchy-Kowalevskaya theorem does not apply directly. But notice that for $t>0, g$ becomes different from 0 , since from the evolution equations we have $g_{t}=4 \Lambda_{1 x} \neq 0$, for all but finitely many $x$. Next we prove:

Lemma 2. The Cauchy problem for the system (14) with the initial data

$$
\left.V(x, y, t)\right|_{t=0}=0
$$

has the unique analytic solution $V \equiv 0$.

Proof. Let us assume that we have analytical solution $V$ of this Cauchy problem. Expand $V$ as well as $U$ in the power series in $t$ :

$$
\begin{gathered}
V(x, y, t)=\sum_{k=0}^{+\infty} W_{k}(x, y) t^{k}, W_{k}(x, y)=\left(\begin{array}{l}
w_{2 k} \\
w_{3 k} \\
w_{4 k}
\end{array}\right), W_{0}(x, y)=\left(\begin{array}{l}
0 \\
0 \\
0
\end{array}\right) \\
\Lambda(x, y, t)=\sum_{k=0}^{+\infty} \lambda_{k}(x, y) t^{k}, \quad \lambda_{0}(x, y)=\Lambda_{1}(x)+\Lambda_{2}(y) \\
u_{0}(x, y, t)=\sum_{k=0}^{+\infty} r_{k}(x, y) t^{k}, \quad r_{0}(x, y)=2 \Lambda_{2}(y)-2 \Lambda_{1}(x) \\
f(x, y, t)=\sum_{k=0}^{+\infty} s_{k}(x, y) t^{k}, \quad s_{0}(x, y)=0 \\
g(x, y, t)=\sum_{k=0}^{+\infty} q_{k}(x, y) t^{k}, \quad q_{0}(x, y)=0 .
\end{gathered}
$$

Let us substitute these expansions into (14). We obtain the following identities:

$$
\begin{gathered}
t q_{1} w_{21}+O\left(t^{2}\right)=0, \\
w_{31}+t\left(2 w_{32}-2 w_{21_{y}}\right)+O\left(t^{2}\right)=0, \\
w_{41}+t\left(2 w_{42}+2 w_{21_{x}}\right)+O\left(t^{2}\right)=0,
\end{gathered}
$$


it then follows that $w_{31}=0, w_{41}=0$. Due to (11) we have

$$
q_{1}=\left.g_{t}\right|_{t=0}=-\left.2 u_{0_{x}}\right|_{t=0}=4 \Lambda_{1_{x}} \neq 0
$$

so $w_{21}=0$ and it means that $W_{1}=0$.

By induction, suppose that $W_{k}(x, y)=0$ for any $k<n$. Substituting the expansion of $V$ into (14) we obtain the following identities:

$$
\begin{gathered}
n t^{n} q_{1} w_{2 n}+O\left(t^{n+1}\right)=0 \\
n t^{n-1} w_{3 n}+O\left(t^{n}\right)=0 \\
n t^{n-1} w_{4 n}+O\left(t^{n}\right)=0
\end{gathered}
$$

and by the same reasons we obtain $w_{2 n}=w_{3 n}=w_{4 n}=0$. So we have $W_{n}=0$. Lemma 2 is proved.

Consider now the metric which we have constructed. We shall use the expansion (16) of $U$ to calculate several first terms of $\Lambda$. Let's substitute the expansions into the system (11). Calculating the corresponding terms, one obtains the following identities:

$$
\begin{gathered}
\lambda_{1}(x, y)=0, \\
\lambda_{2}(x, y)=2\left(\left(\Lambda_{1}^{\prime}(x)\right)^{2}+\left(\Lambda_{2}^{\prime}(y)\right)^{2}\right)+\left(\Lambda_{1}(x)+\Lambda_{2}(y)\right)\left(\Lambda_{1}^{\prime \prime}(x)+\Lambda_{2}^{\prime \prime}(y)\right),
\end{gathered}
$$

it then follows that the metric $\Lambda$ is not Liouville class.

Notice that at the initial moment $t=0$ we have

$$
\frac{\partial \Omega}{\partial t}=\frac{1}{4} \frac{\partial}{\partial t}\left(g_{x}-f_{y}\right)=-\frac{1}{2}\left(u_{0_{x x}}+u_{0_{y y}}\right)=\Lambda_{1}^{\prime \prime}(x)-\Lambda_{2}^{\prime \prime}(y) \neq 0 .
$$

This means that at $t \neq 0$ the nonzero magnetic field appears. Theorem 1 is proved.

\section{Quadratic integrals of magnetic flows}

In this section we prove the following:

Theorem 2. Consider the magnetic flow of the Riemannian metric $d s^{2}=$ $\Lambda\left(d x^{2}+d y^{2}\right)$ with the non-zero magnetic form $\Omega$. Suppose the magnetic flow admits a first integral $F_{2}$ on all energy levels such that $F_{2}$ is quadratic in momenta. Then in some coordinates the functions $\Lambda, \Omega$ have the form of the Example 1, so there exists another integral $F_{1}$ linear in momenta and $F_{2}$ can be written as a combination of $H$ and $F_{1}$. 
Proof. Write the equation $\{F, H\}_{m g}=0$ for all points of the phase space (not restricting to one energy level), where the Hamiltonian $H$ and the integral $F_{2}$ are written as:

$$
H=\frac{1}{2 \Lambda}\left(p_{1}^{2}+p_{2}^{2}\right) ; \quad F_{2}=a_{0} p_{1}^{2}+2 a_{1} p_{1} p_{2}+a_{2} p_{2}^{2}+b_{0} p_{1}+b_{1} p_{2}+c_{0} .
$$

Equating to zero coefficients of monomials $p_{1}^{k} p_{2}^{l}$ we get the following 3 groups (a), (b), (c) of equations depending on the value $k+l=3,2,1$ respectively. The first group (a) is:

$$
\begin{gathered}
\frac{\left(a_{0}\right)_{x}}{\Lambda}-a_{0}\left(\frac{1}{\Lambda}\right)_{x}-a_{1}\left(\frac{1}{\Lambda}\right)_{y}=0 \\
\frac{\left(a_{0}\right)_{y}}{\Lambda}+2 \frac{\left(a_{1}\right)_{x}}{\Lambda}-a_{1}\left(\frac{1}{\Lambda}\right)_{x}-a_{2}\left(\frac{1}{\Lambda}\right)_{y}=0 \\
\frac{\left(a_{2}\right)_{x}}{\Lambda}+2 \frac{\left(a_{1}\right)_{y}}{\Lambda}-a_{0}\left(\frac{1}{\Lambda}\right)_{x}-a_{1}\left(\frac{1}{\Lambda}\right)_{y}=0, \\
\frac{\left(a_{2}\right)_{y}}{\Lambda}-a_{1}\left(\frac{1}{\Lambda}\right)_{x}-a_{2}\left(\frac{1}{\Lambda}\right)_{y}=0 .
\end{gathered}
$$

The first group is the same as for geodesic flow without magnetic field. Therefore in a usual way (following [18]) taking (a1) - (a3) and (a2) - (a4) we arrive to the restrictions $a_{1}=$ const and $a_{0}-a_{2}=$ const. Without loss of generality one can assume $a_{1} \equiv 0, a_{0}-a_{2} \equiv 4$. Then substituting back to the (a1) and (a4) we get

$$
a_{0}=\frac{\phi(y)}{\Lambda}, \quad a_{2}=\frac{\psi(x)}{\Lambda}, \quad 4 \Lambda=\phi(y)-\psi(x) .
$$

The next group of equations (b) reads:

$$
\begin{aligned}
& \frac{\left(b_{0}\right)_{x}}{\Lambda}-b_{0}\left(\frac{1}{2 \Lambda}\right)_{x}-b_{1}\left(\frac{1}{2 \Lambda}\right)_{y}=0 \\
& \frac{\left(b_{1}\right)_{y}}{\Lambda}-b_{0}\left(\frac{1}{2 \Lambda}\right)_{x}-b_{1}\left(\frac{1}{2 \Lambda}\right)_{y}=0 \\
& \frac{\left(b_{1}\right)_{x}}{\Lambda}+\frac{\left(b_{0}\right)_{y}}{\Lambda}-\Omega \frac{2\left(a_{2}-a_{0}\right)}{\Lambda}=0
\end{aligned}
$$


Let us rename $b_{0}=2 f, b_{1}=-2 g$. Then the equations (b1), (b2), (b3) can be written in the form:

$$
\begin{gathered}
f_{x}+\frac{\Lambda_{x}}{2 \Lambda} f-\frac{\Lambda_{y}}{2 \Lambda} g=0, \\
f_{x}+g_{y}=0, \\
\Omega=\frac{1}{4}\left(g_{x}-f_{y}\right) .
\end{gathered}
$$

Using $\left(b 3^{\prime}\right)$ the equations of the last group (c) are :

$$
\begin{aligned}
c_{0_{x}} & =-\frac{1}{2} g\left(g_{x}-f_{y}\right), \\
c_{0_{y}} & =-\frac{1}{2} f\left(g_{x}-f_{y}\right) .
\end{aligned}
$$

One can easily see that using $\left(b 2^{\prime}\right)$ one can rewrite (c1), (c2):

$$
\left(c_{0}+\frac{g^{2}-f^{2}}{4}\right)_{x}-\frac{1}{2}(f g)_{y}=0, \quad\left(c_{0}+\frac{g^{2}-f^{2}}{4}\right)_{y}+\frac{1}{2}(f g)_{x}=0 .
$$

The last equations imply

$$
c_{0}+\frac{g^{2}-f^{2}}{4}=K_{1}, \quad f g=K_{2}
$$

where $K_{1}, K_{2}$ are constants.

Now multiplying $\left(b 1^{\prime}\right)$ by $f \Lambda$ we get the following

$$
\left(f^{2} \Lambda\right)_{x}=K_{2} \Lambda_{y}=K_{2} \frac{\phi^{\prime}(y)}{4}
$$

and analogously for $g$ :

$$
\left(g^{2} \Lambda\right)_{y}=K_{2} \Lambda_{x}=-K_{2} \frac{\psi^{\prime}(x)}{4} .
$$

If the constant $K_{2}$ is not zero, then the last two equations imply that the functions $\phi, \psi$ must be constant since otherwise $f^{2} \Lambda$ or $g^{2} \Lambda$ would be unbounded. Thus $K_{2}=0$. But then we have that

$$
f^{2} \Lambda=F(y), \quad g^{2} \Lambda=G(x)
$$


Multiplying these two and using $f g \equiv 0$ we conclude that

$$
F(y) G(x) \equiv 0
$$

meaning that at least one say $F=f=0$. Then from (b2') we have that

$$
g=g(x)
$$

And we have

$$
g^{2} \Lambda=g^{2}(x) \frac{\phi(y)-\psi(x)}{4}=G(x) .
$$

Therefore the function $\phi(y)$ must be constant. Thus the conformal factor $\Lambda$ must be a function of one variable $\Lambda=\Lambda(x)$, and moreover the magnetic field is $\Omega=\frac{1}{4} g^{\prime}(x)$. In the Example 1 of the Introduction we saw that this is exactly the case when the linear in momenta integral $F_{1}$ exists and it is not hard to verify that the quadratic integral $F_{2}$ can be expressed through $F_{1}$. This completes the proof. 


\section{References}

[1] Agapov S.V.: On the integrable magnetic geodesic flow on a 2-torus. [Russian] Siberian Electronic Mathematical Reports, 12, 868 - 873 (2015).

[2] Bialy M.L.: Rigidity for periodic magnetic fields. Ergod. Theor. Dyn. Syst, 20:6, 1619 - 1626 (2000).

[3] Bialy M.L.: On periodic solutions for a reduction of Benney chain, Nonlinear Differential Equations and Applications, 16, 731 - 743 (2009).

[4] Bialy M.L. and Mironov A.E.: New semi-hamiltonian hierarchy related to integrable magnetic flows on surfaces, Cent. Eur. J. Math., 10:5, 1596 - 1604 (2012).

[5] Bialy M.L. and Mironov A.E.: Rich quasi-linear system for integrable geodesic flow on 2-torus, Discrete and Continuous Dynamical Systems Series A, 29:1, $81-90$ (2011).

[6] Bialy M.L. and Mironov A.E.: Integrable geodesic flows on 2-torus: formal solutions and variational principle, Journal of Geometry and Physics, $\mathbf{8 7 : 1 ,} 39-47$ (2015).

[7] Bialy M.L. and Mironov A.E.: Cubic and quartic integrals for geodesic flow on 2-torus via a system of the hydrodynamic type, Nonlinearity, 24:12, 3541 - 3554 (2011).

[8] Bialy M.L. and Mironov A.E.: From polynomial integrals of Hamiltonian flows to a model of non-linear elasticity, Journal of Differential Equations, 255:10, $3434-3446$ (2013).

[9] Birkhoff G.D.: Dynamical systems, American Mathematical Society Colloquium Publications, Vol. 9, New York (1927).

[10] Bolotin S.V.: First integrals of systems with gyroscopic forces. [Russian] Vestn. Mosk. U. Mat. M+, 6, 75 - 82 (1984).

[11] Bolsinov A.V., Kozlov V.V. and Fomenko A.T.: The Maupertuis principle and geodesic flows on a sphere arising from integrable cases in the dynamics of a rigid body. Russian Mathematical Surveys, 50:3, $473-501$ (1995). 
[12] Bolsinov A.V. and Jovanovic B.: Magnetic geodesic flows on coadjoint orbits. J. Phys. A-Math, 39:16, 247 - 252 (2006).

[13] Burns K. and Matveev V.S.: On the rigidity of magnetic systems with the same magnetic geodesics. P. Am. Math. Soc., 134:2, 427 - 434 (2006).

[14] Denisova N.V. and Kozlov V.V.: Polynomial integrals of geodesic flows on a two-dimensional torus. Russ. Ac. Sc. Sb. Math+, 83:2, 469 - 481 (1995).

[15] Efimov D.I.: The magnetic geodesic flow on a homogeneous symplectic manifold. Siberian Math. J+ 46:1, 83 - 93 (2005).

[16] Greenberg J.M. and Rascle M.: Time-periodic solutions to systems of conservation laws. Arch. Ration. Mech. An., 115:4, 395 - 407 (1991).

[17] John F.: Partial differential equations. Reprint of the fourth edition. Applied Mathematical Sciences, 1. Springer-Verlag, New York, 1991.

[18] Kolokol'tsov V.N.: Geodesic flows on two-dimensional manifolds with an additional first integral that is polynomial in the velocities, Math. USSR Izv,$+ \mathbf{2 1 : 2 ,} 291-306$ (1983).

[19] Kozlov V.V.: Symmetries, topology, and resonances in Hamiltonian mechanics, Springer, Verlag, Berlin. 1996.

[20] Kozlov V.V. and Treschev D.V.: On the integrability of hamiltonian systems with toral position space. Math. USSR Sb+, 63:1, $121-139$ (1989).

[21] Pavlov M.V. and Tsarev S.P.: Tri-hamiltonian structures of Egorov Systems of hydrodynamic type, Funct. Anal. Appl+, 37:1, $32-45$ (2003).

[22] Serre D.: Richness and the classification of quasi-linear hyperbolic systems. Preprint IMA N597, 1989.

[23] Sevennec B.: Geometrie des systemes de lois de conservation, Memories, Soc. Math. de France, Marseille, 56 (1994).

[24] Taimanov I.A.: On an integrable magnetic geodesic flow on the twotorus, Regul. Chaotic Dyn., 20:6, 667 - 678 (2015). 
[25] Ten V.V.: Polynomial first integrals for systems with gyroscopic forces. Math. Notes+, 68:1, $135-138$ (2000).

[26] Tsarev S.P.: On Poisson brackets and one-dimensional hamiltonian systems of hydrodynamic type, Dokl. Math., 31, 488 - 491 (1985).

[27] Tsarev S.P.: The geometry of hamiltonian systems of hydrodynamic type. The generalized hodograph method, Math. USSR Izv+, 37:2, 397 - 419 (1991). 